\title{
Predicting PICU patient survival prognosis: Pediatric Logistic Organ Dysfunction vs Pediatric Index of Mortality scores
}

\author{
Edwina Winiarti, Muhammad Sholeh Kosim, Mohamad Supriatna
}

\begin{abstract}
Background Determining prognosis of patients using scoring systems have been done in many pediatric intensive care units (PICU). The scoring systems frequently used are pediatric logistic organ dysfunction (PELOD), pediatric index of mortality (PIM) and pediatric risk of mortality (PRISM).

Objective To compare the performance of PELOD and PIM scores in predicting the prognosis of survival vs death in PICU patients.

Methods A prognostic test in this prospective, cohort study was conducted in the PICU of the Kariadi General Hospital, Semarang. PELOD and PIM calculations were performed using formulae from previously published articles. Statistical analyses included receiver operating curve (ROC) characteristics to describe discrimination capacity, sensitivity, specificity, positive predictive value, negative predictive value and accuracy.

Results Thirty-three patients fulfilling the inclusion criteria were enrolled in the study. PELOD score for area under the ROC was 0.87 (95\% CI 0.73 to $1.0 ; \mathrm{P}=0.003$ ), while that for PIM was 0.65 (95\% CI 0.39 to $0.90 ; \mathrm{P}=0.2)$. PELOD scores showed sensitivity $85.7 \%$ (95\% CI 59.8 to 100 ), specificity $84.6 \%$ (95\% CI 70.7 to 98.5 ), positive predictive value $60.0 \%$ (95\% CI 29.6 to 90.4 ), negative predictive value $95.6 \%$ (95\% CI 87.3 to 100 ) and accuracy $84.8 \%$. PIM scores showed sensitivity $85.7 \%$ (95\% CI 59.8 to 100 ), specificity $50.0 \%$ (95\% CI 30.8 to 69.2 ), positive predictive value $31.6 \%$ (95\% CI 10,7 to 52.5 ), negative predictive value $92.9 \%$ (95\% CI 79.4 to 100) and accuracy $57.6 \%$.

Conclusion PELOD scoring had better specificity, positive predictive value, negative predictive value, accuracy and discrimination capacity than PIM scoring for predicting the survival prognosis of patients in the PICU. [Paediatr Indones. 2012;52:165-9].
\end{abstract}

Keywords: PELOD score, PIM score, prognosis, PICU

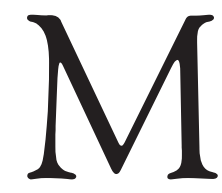
ultiple organ dysfunction syndrome (MODS) is a leading cause of mortality and morbidity in intensive care units. The incidence of MODS in PICUs was reported to be $18-25 \% .{ }^{1}$ Another study reported the incidence to be $11-27 \%^{2}$ and the mortality associated with it was between $26 \%$ and $50 \% .^{3}$ Data from the PICU of Dr. Kariadi General Hospital showed the incidence of MODS to be $35.7 \%$, with a mortality rate of $51.85 \%$ in 2007-2008. Study in Peru found 56.5\% of the PICU patients had MODS, and the mortality was $41.6 \%$ in children with MODS. ${ }^{3}$

MODS diagnosis is confirmed by observation of severely ill patients who experience dysfunction of at least two organs simultaneously. These organs include the respiratory, cardiovascular, neurological, hematological, renal, hepatic, and gastrointestinal systems. ${ }^{4}$ The criteria to diagnose organ dysfunction in infants and children were defined by Proulx et al. in $1996^{5}$ and updated in 2005 by Goldstein et al. ${ }^{6}$

From the Department of Child Health, Diponegoro University, Medical School, Dr. Kariadi Hospital, Semarang, Indonesia.

Reprint requests to: Edwina Winiarti. Department of Pediatrics, Medical School, Diponegoro University, Dr. Kariadi Hospital, Semarang, Jl. Dr. Soetomo no. 18, Semarang. E-mail: winnydr@gmail.com 
Measurement of organ dysfunction/failure is important for several reasons: to limit the severity of illness in different intensive care units and patient groups, to make comparisons between time periods or between groups, for use in clinical trials, to classify patients at admission, to compare treatment groups and to evaluate the effects of experimental therapies and procedures on morbidity. ${ }^{2}$

Two prognostic scores have commonly been used to estimate the severity of disease in critically ill patients. The PIM score has been used to describe the illness severity of critically ill patients on admission. This score assesses several comorbidity factors and physiological disorders upon admission to the PICU, or randomly in clinical trials. The PIM score was developed to maximize risk-of-death predictions among some groups of critically ill patients, based on their degree of illness. The PELOD score has also been used to describe the illness severity of critically ill patients during treatment in the PICU. ${ }^{4}$

We aimed to compare PELOD and PIM scores with prognoses of patients who were treated in the PICU of Dr. Kariadi Hospital, Semarang.

\section{Methods}

The research was conducted at the PICU of Dr. Kariadi Hospital, Semarang, from March to July 2010. This prognostic study had a prospective cohort design to compare PELOD and PIM scores in determining the prognosis of patients treated in the PICU. Subjects were children with severe illness requiring treatment in the PICU. We included children who had at least certain laboratory test results (leukocytes, platelets, AST, creatinine, prothrombin time and blood gas analyses) and with sufficient available data to assess their PELOD and PIM scores. Subjects' parents provided written informed consent. We excluded patients who went home before completing the PICU treatment or whose conditions could not be traced at home. PELOD and PIM scores were assessed at baseline, within the first 24 hours of PICU admission. PIM and PELOD scores were measured once by researchers with the assistance of PICU doctors. Subjects were followed as to whether they survived or died.

Data analyses included descriptive analysis and hypothesis test (Mann-Whitney and Fisher-exact tests). The abilities of PIM and PELOD scores to discriminate prognoses of severely ill children was analyzed using ROC curves. The sensitivity, specificity, positive predictive value, negative predictive value and accuracy of each score were calculated by 2 X 2 tables. We used SPSS for Windows v.15.0 (SPSS Inc. USA) to analyze the data. $P$ values of $<0.05$ were considered significant within the $95 \%$ confidence interval range. This study was approved by the Director of Dr. Kariadi General Hospital and the Commission on Health Research Ethics, Faculty of Medicine, Diponegoro University / Dr. Kariadi General Hospital, Semarang.

Table 1. Characteristics of subjects based on discharge conditions from the PICU

\begin{tabular}{|c|c|c|c|}
\hline \multirow[b]{2}{*}{ Characteristics } & \multicolumn{2}{|c|}{ Discharge condition from PICU } & \multirow[b]{2}{*}{$\mathrm{P}$} \\
\hline & $\begin{array}{c}\text { Non-survivors } \\
\mathrm{n}=7\end{array}$ & $\begin{array}{c}\text { Survivors } \\
n=26\end{array}$ & \\
\hline Mean age, months \pm SD & $35.6 \pm 38.42$ & $41.3 \pm 42.63$ & $0.8^{¥}$ \\
\hline \multicolumn{4}{|l|}{ Sex, n (\%) } \\
\hline - Male & $3(42.9)$ & $11(42.3)$ & \\
\hline - Female & $4(57.1)$ & $15(57.7)$ & $0.6 \S$ \\
\hline \multicolumn{4}{|l|}{ Nutritional status, n (\%) } \\
\hline - Severe-moderate malnutrition & $0(0)$ & $10(38.5)$ & \\
\hline - Normal-overweight & $7(100)$ & $16(61.5)$ & $0.06^{\S}$ \\
\hline \multicolumn{4}{|l|}{ Cause of PICU admission, $\mathrm{n}(\%)$} \\
\hline - Surgical & $1(14.3)$ & $7(26.9)$ & \\
\hline - Non-surgical & $6(85.7)$ & $19(73.1)$ & $0.6^{\S}$ \\
\hline \multicolumn{4}{|l|}{ Respiratory distress, n (\%) } \\
\hline - Yes & $6(85.7)$ & $13(50.0)$ & \\
\hline - No & $1(14.3)$ & $13(50.0)$ & $0.2^{\S}$ \\
\hline
\end{tabular}

$¥$ Mann-Whitney test; § Fisher-exact test 
Edwina Winiarti et al: PICU patient survival prognosis: PELOD vs PIM scores

Table 2. Discrimination capacity and diagnostic value of PELOD and PIM scores for PICU survival

\begin{tabular}{lcc}
\hline & PELOD & PIM \\
\hline Area under ROC $(95 \% \mathrm{Cl})$ & $0.87(0.73$ to 1.0$)$ & $0.65(0.39$ to 0.90$)$ \\
Sensitivity, \% $(95 \% \mathrm{Cl})$ & $85.7(59.8$ to 100$)$ & $85.7(59.8$ to 100$)$ \\
Specificity, \% $(95 \% \mathrm{Cl})$ & $84.6(70.7$ to 98.5$)$ & $50.0(30.8$ to 69.2$)$ \\
Positive predictive value, \% $(95 \% \mathrm{Cl})$ & $60.0(29.6$ to 90.4$)$ & $31.6(10.7$ to 52.5$)$ \\
Negative predictive value, \% $(95 \% \mathrm{Cl})$ & $95.6(87.3$ to 100$)$ & $92.9(79.4$ to 100$)$ \\
Accuracy, \% & 84.8 & 57.6 \\
\hline
\end{tabular}

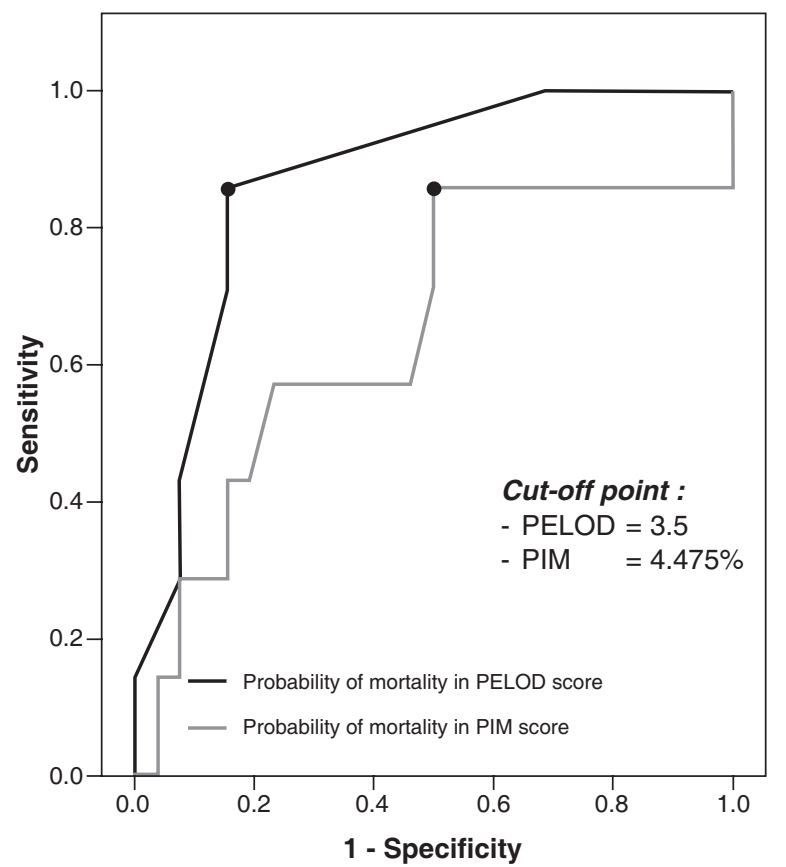

Area under ROC curve :

- PELOD score $=0.87(95 \% \mathrm{Cl}=0.73$ to $1.0 ; \mathrm{P}=0.003)$

- PIM score $\quad=0.65(95 \% \mathrm{Cl}=0.39$ to $0.90 ; \mathrm{P}=0.2)$

Figure 1. ROC analysis for predicting survival outcomes based on PELOD and PIM scores $(n=33)$

\section{Results}

There were 33 subjects in our study, consisting of 14 males $(42.4 \%)$ and 19 females $(57.5 \%)$. There were no significant differences in sex, age, nutritional status, cause of PICU admission or presence of respiratory distress in patients who survived or died. Subjects' characteristics based on their discharge conditions from the PICU (died vs survived) are shown in Table 1.
The ROC analysis of PELOD and PIM scores to predict outcomes is shown in Figure 1.

The area under the ROC for the PELOD score to predict discharge condition from the PICU was 0.87 (95\% CI 0.73 to $1.0 ; \mathrm{P}=0.003$ ) with a cut-off point of 3.5. The area under the ROC curve for the PIM score was 0.65 (95\% CI 0.39 to $0.90 ; \mathrm{P}=0.2)$ with a cut-off point of $4.47 \%$.

Diagnostic test results indicated the sensitivity of the PELOD score to be $85.7 \%$, the same as the PIM score's sensitivity (85.7\%). The specificity of the PELOD score was better than that of the PIM score $(84.6 \%$ vs $50.0 \%$, respectively). The accuracy of the PELOD score was also better than that of the PIM score ( $84.8 \%$ vs $57.6 \%$, respectively) as shown in Table 2.

\section{Discussion}

In our study, 7 out of 33 subjects died, similar to the results of Qureshi et al. ${ }^{7}$ and Marlina et al. ${ }^{8}$ (28.7\% and $28.3 \%$, respectively). In contrast, Leteurtre et al. ${ }^{9}$ reported that only $6.4 \%$ of patients died in their study on PELOD scoring. Qureshi et al. stated that the causes of poor performance were the healthcare system, different cases, different patterns of disease, higher number of non-surgical patients (in this study 25 out of 33 subjects), length of illness, history of treatment before PICU admission, level of clinical instability while being treated and severity of disease when the patients first came to the hospital. ${ }^{7}$

We found the PELOD to give good discrimination, as assessed by ROC curve. The PELOD discrimination was 0.87 (95\% CI 0.73 to 1.0 ). Discrimination is considered excellent if the ROC area is $>0.9$, good for 0.80 to 0.90 , and fair for 0.70 to $0.80 . .^{7}$ PIM score had poor discrimination because the area under ROC curve was 0.65 (95\% CI 0.39 to 0.90). Our results were 
consistent with the study by Thukral et al. in Northern India, in which the area under the PELOD ROC was good (0.80), mortality was directly related to the degree of organ dysfunction and PELOD scores increased with increasing number of dysfunctional organs. ${ }^{5}$ In a comparison of PRISM III and PELOD scores as a predictor of mortality in patients with dengue shock syndrome, Iskandar et al. from the PICU at Harapan Kita Hospital, Jakarta, showed that PELOD score could better predict mortality (ROC 0.953) than PRISM III scores (ROC 0.889). ${ }^{10}$

Different results were obtained by Qureshi et al. in a comparison of PRISM, PIM2 and PELOD scores to predict mortality. ${ }^{7}$ Their study showed that PIM2 had better discrimination (ROC 0.88) compared to PRISM (ROC 0.78) and PELOD (ROC 0.77). Calibration of PRISM was better $(\mathrm{P}=0.49)$ compared to PIM2 $(\mathrm{P}=0.29)$ and PELOD $(\mathrm{P}<0.01)$. Furthermore, Marlina et al. in Bandung, Indonesia also reported better discrimination for PIM2 (ROC 0.783) compared to PELOD scores (ROC 0.706). ${ }^{8}$ Better performance of PIM2 test may have been due to differences in demographic profiles, distribution of disease or the availability of infrastructure, including trained personnel and equipment. In addition, similar illness severity may result in different outcomes due to different pathological processes. ${ }^{7}$

Nutritional status also affects physiological responses and outcomes. The prevalence of acute and/or chronic malnutrition in children treated in PICU was estimated to be $24 \% .{ }^{11}$ We found that $8 / 33$ of subjects had malnutrition, $2 / 33$ had poor nutrition, $2 / 33$ were overweight but most had good nutrition (21 out of 33). All patients who died had either good nutrition or were overweight. In contrast, Qureshi et al. in Pakistan reported that most patients suffered from malnutrition, ${ }^{7}$ and Marlina et al. reported that $64.7 \%$ of patients who died suffered from malnutrition. ${ }^{8}$ We observed that no subjects with malnutrition or poor nutrition died. However, test results showed there was no statistically significant difference between the survivor and non-survivor groups $(\mathrm{P}=0.06)$, therefore, malnutrition could not be used as a prognostic factor. We also had fewer patients with malnutrition and poor nutrition (10 out of 33 subjects) than patients who were well-nourished or overweight. Hence, cause of death was more influenced by severity of illness, comorbid conditions and underlying diseases. $1,3,5,7,9,12$
A limitation of our study was that we did not distinguish the type of disease affecting prognosis. Another limitation was that patient assessment was performed only at the time of patient discharge from the PICU. Prediction of mortality and morbidity following PICU treatment needed to be further investigated.

In conclusion, PELOD test was better than PIM test for predicting the survivability prognosis of children treated in the PICU. PELOD score should be calculated for every newly admitted PICU patient.

\section{References}

1. Leclerc F, Leteurtre S, Duhamel A, Grandbastien B, Proulx F, Martinot A. Cumulative influence of organ dysfunction and septic state on mortality of critically ill children. Am J Respir Crit Care Med. 2005;171:348-53.

2. Peres Bota D, Melot C, Lopes Ferreira F, Nguyen Ba V, Vincent JL. The multiple organ dysfunction score (MODS) versus the sequential organ failure assessment (SOFA) score in outcome prediction. Intensive Care Med. 2002;28:1619-24.

3. Tantalean JA, Leon RJ, Santos AA, Sanchez E . Multiple organ dysfunction syndrome in children. Pediatr Crit Care Med. 2003;4:181-5.

4. Lacroix J, Cotting J, for the Pediatric Acute Lung injury and Sepsis Investigators (PALISI) Network. Severity of illness and organ dysfunction scoring in children. Pediatr Crit Care Med. 2005;6:S126-34.

5. Proulx F, Fayon M, Farrel CA, Lacroix J, Gauthier M. Epidemiology of sepsis and multiple organ dysfunction syndrome in children. Chest. 1996;109:1033-7.

6. Brilli RJ, Goldstein B. Pediatric sepsis definitions: past, present, and future. Pediatr Crit Care Med. 2005;6:S6-8.

7. Qureshi AU, Ali AS, Ahmad TM. Comparison of three prognostic scores (PRISM, PELOD and PIM 2) at pediatric intensive care unit under Pakistani circumstances. J Ayub Med Coll Abbottabad. 2007;19:49-53.

8. Marlina L, Somasetia DH, Garna H. Perbandingan penggunaan pediatric index of mortality 2 (PIM2) dan skor pediatric logistic organ dysfunction (PELOD) untuk memprediksi kematian pasien sakit kritis pada anak. Sari Pediatri. 2008;10:262-7.

9. Leteurtre S, Martinot A, Duhamel A, Proulx F, Grandbastien B, Cotting J, et al. Validation of the paediatric logistic organ dysfunction (PELOD) score: prospective, observational, multicentre study. Lancet. 2003;362:192-7. 
Edwina Winiarti et al: PICU patient survival prognosis: PELOD vs PIM scores

10. Iskandar HR, Mulyo D, Agnes P, Suryatin Y. Comparison of Pediatric Logistic Organ Dysfunction (PELOD) and Pediatric Risk of Mortality (PRISM) III scores as a mortality predictor in pediatric dengue shock syndrome (DSS) patients. Pediatrics. 2008;121;129.

11. Mehta N, Castillo L. Nutrition in the critically ill child. In:
Fuhrman BP, Zimmerman JJ, editors. Pediatric critical care. $3^{\text {rd }}$ ed. Philadelphia: Mosby Elsevier; 2006. p. 1068-83.

12. Han YY, Shanley TP. Multiple organ dysfunction syndrome. In: Nichols DG, ed. Rogers' textbook of pediatric intensive care. Philadelphia: Wolters Kluwer/Lippincott Williams \& Wilkins; 2008. p. 283-99. 